\title{
Razones Que Impulsan La Motivación De Compra De Los Estudiantes Universitarios Mexicanos
}

\author{
Dra. Laura Fischer \\ Facultad de Contaduría y Administración, \\ Universidad Nacional Autónoma de México \\ Mtro. Jorge Espejo \\ Universidad Autónoma del Estado de Morelos
}

doi: 10.19044/esj.2016.v12n7p275 URL:http://dx.doi.org/10.19044/esj.2016.v12n7p275

\begin{abstract}
In order to make a person interested and decided to purchase a product or service it is necessary to have something triggering the decision: an innate or absolute need; a relational need or a originated the marketing efforts done by the supplier. We present in this paper the results of an explorative, descriptive and correlational research. Considering a quantitative approach based on the reasons that motivates an individual to purchase proposed by Eisenberg (Bryaneisenberg, 2015).The research comprises a sample of 2400 university students from México in order to know the most influential reasons for purchasing among the chosen segment. Results show that the marketing efforts are the most influentional reasons for purchasing
\end{abstract}

Keywords: Purchase motivation, needs

\section{Resumen}

Para que una persona se interese y decida la compra de un producto o servicio debe existir un detonante que lo impulse: una necesidad innata o absoluta, una necesidad relativa o una necesidad fomentada por los esfuerzos mercadológicos que realizan las empresas. En este estudio, se realizó un estudio exploratorio, descriptivo y correlacional con enfoque cuantitativo que tomó como referencia las razones propuestas por Eisenberg (Bryaneisenberg 2015) por las que un individuo es motivado a realizar una compra. El estudio se aplicó a una muestra de 2400 estudiantes universitarios de la República Mexicana con el objetivo de conocer los factores de mayor influencia en las motivaciones de compra de ese segmento. Los resultados muestran que las estrategias planteadas en mercadotecnia son los factores importantes en la motivación de compra. 
Palabras claves: Motivación de compra, necesidades

\section{Introducción}

La motivación de compra en el consumidor surge con el reconocimiento de una discrepancia entre una situación actual y una situación deseada. El resultado de esta discrepancia es una necesidad de cambio - una necesidad que a veces es aparente y otras veces más latente - y casi siempre aparece cuando el consumidor enfrenta por primera vez productos innovadores (Feldman, 2003).

Para que una persona se interese en la compra de un producto o servicio, debe estar motivada por el uso o consumo del mismo. Para Arana, Meilán, Gordillo y Carro (2010), la motivación es esa fuerza que impulsa la conducta de las personas a cumplir con objetivos. Es importante considerar que la fuerza impulsora no aparece de forma espontánea, siempre habrá razones que surgen de las necesidades personales transformadas en deseos para que esto ocurra; los clientes consumen productos o servicios primeramente por el surgimiento de necesidades, que al manifestarse se transforman en deseos, provocando así la aparición de motivaciones de compra. En la comercialización de sus productos y servicios, las empresas deben estimular a los clientes y es particularmente importante que durante el periodo de transición del consumidor, de la situación actual a la situación deseada, la empresa procure la aparición de fuerzas de impulso irresistibles o de peso para que los consumidores adquieran el bien o servicio de su marca.

\section{Marco teórico}

La motivación es un proceso básico que involucra el mantenimiento o la mejora de la vida del individuo (Petri, 1991), también se utiliza para indicar la dirección selectiva de una conducta (Cacciopo y Berntson 1992, citado por Palmero, Fernández- Abascal, Martínez, Chóliz. 2002 p.37). Los psicólogos que estudian la motivación buscan descubrir las metas particulares deseadas, es decir los motivos que subyacen al comportamiento (Deckers, 2001). Dichos motivos pueden ejemplificarse con comportamientos tan básicos como beber para satisfacer la sed, o tan triviales como caminar para hacer ejercicio (Feldman, 2003). En este sentido, el estudio de la motivación consiste en explicar por qué las personas buscan hacer determinadas cosas.

La motivación puede definirse como el proceso de estimular a un individuo para que se realice una acción que satisfaga alguna de sus necesidades, deseos y expectativas (Lamb, Hair y McDaniel 2002 y Sexton, 1977). Según Stoner (Stoner, 1996) la motivación es una característica de la psicología humana, que incluye los factores que ocasionan, canalizan y 
sostienen la conducta humana, ya que interviene una serie de factores psicológicos como la personalidad de los individuos, su autorrealización, el reconocimiento que tienen, el logro y los cumplimientos que asumen, y la autoeficacia que percibe sobre sí mismos.

La motivación en el consumidor está ligada a las necesidades, encontrando dos tipos de necesidades: (a) las necesidades innatas o absolutas, que son naturales, inherentes al organismo humano, (Maslow 1954; Maslow, y Hoffman, 1996) y (b) las necesidades relativas que se dan como necesidades culturales, sociales, que dependen de la experiencia, de las condiciones ambientales y la evolución de la sociedad (Lambin, Gallucci y Sicurello 2009).

Dentro de las necesidades innatas o absolutas, existen factores personales o también considerados de imagen propia, como son: la salud, la belleza, el estado físico y de equilibrio (homeostasis). Cuando se percibe el producto o servicio como medio para mejorar la imagen propia, se vuelve más fuerte y es probable que se convierta en un factor más duradero, que funcione como un rasgo estable de motivación de compra (Molla, 2006) y (Blackwell, 2002).

En relación a las necesidades relativas destaca el estilo de vida de una persona expresado en sus actitudes, intereses y opiniones, es algo más que la clase social o la personalidad; es todo un patrón de acción e interacción con el mundo, denota por completo a la persona en interacción con su ambiente (Ferre, 1997), (Ruiz, 2006).

Existen las estrategias o esfuerzos mercadológicos que buscan posicionar sus productos en la mente de los consumidores y aunque no se puede crear necesidades innatas preexistentes, inherentes al ser humano, éstas se encuentran latentes en el individuo aunque no está enterado o no existen en su conciencia (Kotler, 2000). Una crítica fuerte que se le ha dado a la mercadotecnia es que ha transformado al mercado en un mecanismo que crea necesidades en lugar de satisfacerlas. Para Attali y Guillaume (Attali, 1974) los productores explotan la dinámica de los deseos para encontrar mercados que les permitan preservar su poder económico, asimismo de la Rosa (Rosa, 1977), manifiesta que existen necesidades reales y necesidades falsas, creación de la sociedad y del productor.

Bryan Eisenberg (Bryaneisenberg 2015) argumenta que puesto que el deber de las marcas es conocer a sus clientes, es también su deber estar al tanto de sus diferentes motivaciones de compra. Menciona que durante la compra de un producto o servicio influye una pluralidad de factores, con este postulado, planteó 20 razones (Tabla 1), que enfrenta el consumidor a la hora de decidirse por la compra de uno u otro producto. 
Tabla 1 Veinte razones que motivan la compra (Bryaneisenberg 2015)

\begin{tabular}{|c|c|c|}
\hline Din & Variable & Ítem \\
\hline $\begin{array}{l}\text { Necesidades } \\
\text { innatas o } \\
\text { absolutas }\end{array}$ & $\begin{array}{l}\text { V.1 Necesidades } \\
\text { básicas } \\
\text { V.2 Conveniencia } \\
\text { V.3 Recambio } \\
\text { V.11 Compra } \\
\text { obligatoria } \\
\text { V.18 Adicción } \\
\text { V.19 } \\
\text { Miedo/Seguridad }\end{array}$ & $\begin{array}{l}\text { 1. Se tiene inevitablemente que llenar la nevera. } \\
\text { 2. Se necesita algo urgentemente en un momento } \\
\text { concreto y se compra lo que está más a mano } \\
\text { 3. A veces hay que comprar algo para sustituir otra } \\
\text { cosa que ya no da el servicio que se espera de } \\
\text { ella } \\
\text { 11. Una fuerza externa obliga al consumidor a } \\
\text { comprar un } \\
\text { producto } \\
\text { 18. Una fuerte adicción motiva la compra } \\
\text { 19. Compra algo simplemente para sentirse más } \\
\text { seguro }\end{array}$ \\
\hline $\begin{array}{r}\text { Nece } \\
\text { rel }\end{array}$ & $\begin{array}{c}\text { V.5 Prestigio } \\
\text { V.6 Vacío } \\
\text { emocional } \\
\text { V.12 Alimento } \\
\text { para el ego } \\
\text { V.13 } \\
\text { Identificación } \\
\text { V.14 Presión } \\
\text { V.16 Reciprocidad } \\
\text { V.20 Indulgencia }\end{array}$ & $\begin{array}{l}\text { 5. Se compra un producto para ganar en "prestigio" } \\
\text { de cara a los demás. } \\
\text { 6. La gente compra simplemente para sustituir } \\
\text { cosas que ni tiene ni nunca tendrá } \\
\text { 12. El consumidor compra simplemente un producto } \\
\text { porque cree que éste le va ayudar a sentirse } \\
\text { mejor que el resto } \\
\text { 13. El consumidor compra porque se siente } \\
\text { identificado con el target del producto } \\
\text { 14. La gente compra lo que tienen también sus } \\
\text { amigos } \\
\text { 16. Se compra un producto para dárselo a otra } \\
\text { persona que previamente le ha dado algo. } \\
\text { 20. Un producto se compra simplemente por darse } \\
\text { el gusto de hacerlo }\end{array}$ \\
\hline $\begin{array}{c}\text { Esfuerzos } \\
\text { mercadológico }\end{array}$ & $\begin{array}{l}\text { V.4 Escasez } \\
\text { V.7 Precios bajos } \\
\text { V.8 Buena } \\
\text { relación } \\
\text { calidad - } \\
\text { precio } \\
\text { V.9 } \\
\text { Reconocimien } \\
\text { to del nombre } \\
\text { de la marca } \\
\text { V.10 Moda e } \\
\text { innovación } \\
\text { V.15 Solidaridad } \\
\text { V.17 Empatía }\end{array}$ & $\begin{array}{l}\text { 4. Se compra un producto por su exclusividad o } \\
\text { porque sus existencias son ya muy limitadas. } \\
\text { 7. Un producto a un precio excepcionalmente bajo } \\
\text { es casi siempre un imán para el consumidor. } \\
\text { 8. No se necesita en realidad el producto, pero lo } \\
\text { termina comprando porque su relación calidad- } \\
\text { precio es inmejorable } \\
\text { 9. Cuando compra por primera vez un determinado } \\
\text { producto, el consumidor se suele decantar por la } \\
\text { marca más conocida, aquella que le resulta más } \\
\text { familiar. } \\
\text { 10. El consumidor compra un producto porque está } \\
\text { de moda y todo el mundo habla de él. } \\
\text { 15. Se compra un producto porque se cree que } \\
\text { haciéndolo está contribuyendo a una buena } \\
\quad \text { causa } \\
\text { 17. Compra un producto porque siente empatía por } \\
\text { él, aun siendo consciente de que su relación } \\
\text { calidad-precio no es la mejor }\end{array}$ \\
\hline
\end{tabular}


Es una realidad que para el individuo, en algún momento de su comportamiento, el motivador de compra de cierto producto o servicio se reflejará en una o varias de las razones expuestas en la tabla 1, pero ¿cuál o cuáles de estas razones influyen más en el comportamiento de compra del estudiante universitario mexicano?, ¿a qué característica le dan mayor peso? Resolver estas cuestiones permitirá identificar con mayor precisión las variables más influyentes en la compra.

Para dar respuesta a este cuestionamiento se realizó una investigación que tomó como referencia las 20 razones de Eisenberg (Bryaneisenberg 2015) por las que un individuo es motivado a realizar una compra. Las 20 razones se clasificaron en necesidades innatas o absolutas, relativas y de esfuerzo mercadológico, (tabla 1) con el objetivo de conocer los factores de mayor influencia en las motivaciones de compra de los estudiantes universitarios mexicanos.

Pera responder este objetivo se planteó la siguiente hipótesis:

$H_{1}=$ Los factores de mayor influencia en la motivación de compra de los estudiantes universitarios mexicanos son los creados o fomentados por los esfuerzos mercadológicos

\section{Método}

Se realizó un estudio exploratorio, descriptivo y correlacional con enfoque cuantitativo, basado en un cuestionario estructurado. Dicho cuestionario fue probado en quince estudiantes pertenecientes a la población objetivo y tuvo por resultado correcciones en la redacción de algunas de las preguntas y el nombramiento de las variables. En el cuestionario final, de 20 ítems, se utilizaron las 20 motivaciones de compra propuestas por Eisenberg (Bryaneisenberg 2015) en una escala tipo Likert de 4 puntos (1 nunca; 2 pocas veces; 3 casi siempre o 4 siempre). El trabajo de campo fue llevado a cabo durante los meses de Marzo y Abril del año 2015. El método de levantamiento de datos fue el de cuestionarios electrónicos asistidos por computadora en forma remota (Surveymonkey). El estudio tomó como plataforma las 180 universidades e instituciones de educación superior en el país, registradas en la Asociación Nacional de Universidades e Instituciones de Educación Superior en México, (ANUIES 2015), con una población de tres millones quinientos mil alumnos (Red académica 2013). La investigación fue realizada a partir de una muestra de 2,426 estudiantes universitarios compradores a nivel nacional (error de estimación $2 \%$ y nivel de confianza 95\%). Después de analizar la existencia de datos ausentes y casos atípicos, la muestra finalmente utilizada fue de $2400^{8}$ estudiantes

\footnotetext{
${ }^{8}$ Agradecemos la participación de las Instituciones de Nivel Superior de los Estados, Aguascalientes, Baja California Norte, Campeche, Colima, Chihuahua, Chiapas, Coahuila,
} 
universitarios compradores (error de estimación $=+/-2 \%$ y nivel de confianza 95\%). La tabla 2 muestra la ficha técnica que resume las características de la investigación realizada.

Tabla 2. Ficha técnica (elaboración propia)

\begin{tabular}{|c|c|}
\hline Universo & $3,500,000$ alumnos universitarios \\
\hline Alcance de la investigación & Nacional \\
\hline Unidad muestral & Estudiantes universitarios compradores \\
\hline Método de levantamiento de la información & $\begin{array}{c}\text { Cuestionarios electrónicos asistidos por } \\
\text { computadora en forma remota } \\
\text { (Surveymonkey) }\end{array}$ \\
\hline Procedimiento de muestreo & Aleatorio \\
\hline Tamaño de la muestra & 2,400 cuestionarios aplicados \\
\hline Margen de error de muestreo & $\begin{array}{c}\text { Para un nivel de confianza del } 95 \% \text { (p/q }= \\
\text { 0.5), y datos referidos a estudiantes } \\
\text { universitarios compradores: }+/-2 \%\end{array}$ \\
\hline Fecha del trabajo de campo & Del 1 de Marzo al 30 de abril de 2015 \\
\hline
\end{tabular}

\section{Análisis}

En primer lugar, se examinó la consistencia interna del cuestionario, así como de cada una de las escalas, utilizando para ello los estadísticos alpha de Cronbach (Cronbach, 1951) y fiabilidad par-impar. A continuación se realizó un análisis descriptivo de las 20 motivaciones de compra propuestas por Eisenberg (Bryaneisenberg 2015); finalmente se realizó un análisis de regresión lineal múltiple, el que permitió determinar la importancia de las diferentes motivaciones de compra.

\section{Consistencia interna del cuestionario}

Los estadísticos alpha de Cronbach (Cronbach, 1951) y Fiabilidad par-impar reportaron: .844 y .831 respectivamente, nivel "bueno" conforme a la escala recomendada por George y Mallery (1995). Se puede concluir, a partir de los valores obtenidos, que los 20 ítems están correlacionados positivamente unos con otros pues miden en cierto grado una entidad en común.

\section{Análisis descriptivo}

El análisis descriptivo de la muestra identifica cuáles son las principales motivaciones de compra de los estudiantes universitarios en México. En la tabla 3 se puede observar que por arriba del promedio del valor de Likert (2.307) predominan los esfuerzos mercadológicos como

Distrito Federal, Estado de México, Guanajuato, Guerrero, Hidalgo, Jalisco, Michoacán, Morelos, Nayarit, Oaxaca, Puebla, Querétaro, San Luis Potosí, Sinaloa, Sonora, Tabasco, Tamaulipas, Tlaxcala, Veracruz, Yucatán, Zacatecas 
principales motivadores de compra, mostrando el valor Likert promedio más alto (2.473)

Tabla 3. Valor Likert de los motivadores de compra reportados por los estudiantes universitarios en México (elaboración propia)

\begin{tabular}{|c|c|c|c|}
\hline \multicolumn{2}{|c|}{ Dimensión } & Variable & Valor Likert $^{9}$ \\
\hline 1 & Necesidad absoluta & V.1 Necesidades básicas & 3.498 \\
\hline 3 & Esfuerzo mercadológico & V.8 Buena relación calidad - precio & 3.248 \\
\hline 1 & Necesidad absoluta & V.3 Recambio & 3.095 \\
\hline 3 & Esfuerzo mercadológico & V.9 Reconocimiento del nombre de la & 2.829 \\
\hline 2 & mecesidad relativa & V.16 Reciprocidad & 2.634 \\
\hline 2 & Necesidad relativa & V.5 Prestigio & 2.532 \\
\hline 2 & Necesidad relativa & V.20 Indulgencia & 2.526 \\
\hline 3 & Esfuerzo mercadológico & V.4 Escasez/Exclusividad & 2.424 \\
\hline 3 & Esfuerzo mercadológico & V.17 Empatía & 2.314 \\
\hline 3 & Esfuerzo mercadológico & V.7 Precios bajos & 2.299 \\
\hline 1 & Necesidad absoluta & V.2 Conveniencia & 2.259 \\
\hline 3 & Esfuerzo mercadológico & V.10 Moda e innovación & 2.158 \\
\hline 3 & Esfuerzo mercadológico & V.15 Solidaridad & 2.042 \\
\hline 2 & Necesidad relativa & V.6 Vacío emocional & 1.984 \\
\hline 2 & Necesidad relativa & V.13 Identificación & 1.963 \\
\hline 1 & Necesidad absoluta & V.18 Adicción & 1.877 \\
\hline 2 & Necesidad relativa & V.12 Alimento para el ego & 1.751 \\
\hline 1 & Necesidad absoluta & V.19 Miedo/Seguridad & 1.655 \\
\hline 2 & Necesidad relativa & V.14 Presión & 1.545 \\
\hline 1 & Necesidad absoluta & V.11 Compra obligatoria & 1.507 \\
\hline & & Promedio general & 2.307 \\
\hline & & Promedio Necesidades absolutas & 2.315 \\
\hline & & Promedio Necesidades relativas & 2.134 \\
\hline & & & 2.473 \\
\hline
\end{tabular}

La tabla 4 muestra los valores Likert por género y edad, su estructura es muy similar al patrón mostrado en la tabla 3. Comparando los promedios parciales presentados en la tabla 4 con los promedios totales mostrados en la tabla 3 se pueden observar las siguientes expresiones motivacionales de los estudiantes universitarios en México:

\footnotetext{
${ }^{9}$ Promedio en 2400 encuestas aplicadas a nivel nacional
} 
Tabla 4. Valores Likert para los motivadores de compra por Género y Edad (elaboración propia)

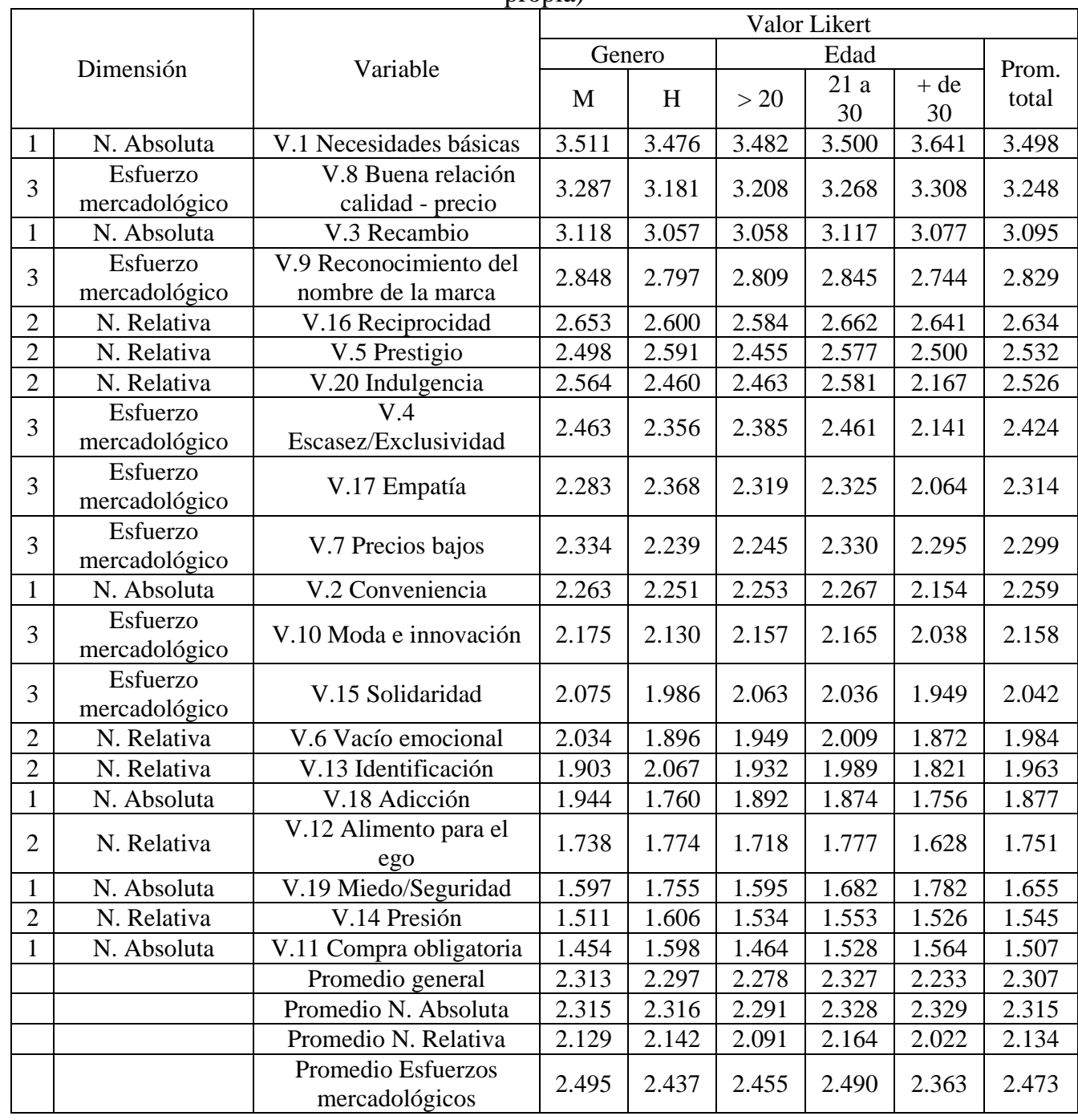

(a) Los estudiantes mayores de 21 años son motivados a comprar prioritariamente por sus necesidades innatas o absolutas, independientemente de su género: 2.328 y 2.329 vs. 2.315

(b) La motivación de compra a partir de necesidades relativas se presenta en los estudiantes de educación superior en México entre los 21 y 30 años de edad en ambos géneros: 2.164 vs. 2.134

(c) Los esfuerzos mercadológicos son los principales motivadores de compra en los estudiantes universitarios en México, después los motivan las necesidades absolutas y finalmente las necesidades relativas: 2.473 vs. 2.315 y 2.134 respectivamente. Esto confirma la hipótesis central planteada para esta investigación 
(d) Los esfuerzos mercadológicos ejercen mayor influencia en los estudiantes entre 21 y 30 años de edad, principalmente en el sexo femenino: 2.473 vs. 2.490 y 2.495 respectivamente.

\section{Análisis de regresión lineal múltiple}

Tras efectuar el análisis descriptivo de la muestra, se procedió a determinar la importancia de las motivaciones de compra a través de un análisis de regresión múltiple, siendo la variable dependiente un factor de motivación central, determinado a partir del promedio motivacional registrado en cada una de las observaciones. La tabla 5 muestra la importancia de cada ítem de motivación en las tres dimensiones. En la misma tabla se muestran, para cada una de las dimensiones, los coeficientes de determinación ajustados: $\mathrm{R}^{2}$ (necesidades innatas $\mathrm{o}$ absolutas $=67.2 \%$, necesidades relativas $=81.8 \%$ y esfuerzos mercadológicos $=74.9 \%$ ) el nivel de los coeficientes indica un grado de fidelidad aceptable en la pauta de relación existente en los datos recolectados.

Tabla 5. Importancia de los motivadores de compra (elaboración propia MINITAB)

\begin{tabular}{|c|c|c|c|}
\hline Dimensión & Ítem & Coef. & $\mathrm{P}$ \\
\hline $\begin{array}{c}\text { Necesidades innatas } \\
\text { o absolutas } \\
\mathrm{R}^{2}=67.2 \%\end{array}$ & $\begin{array}{l}\text { 11. Una fuerza externa obliga al } \\
\text { consumidor a comprar un } \\
\text { producto } \\
\text { 18. Una fuerte adicción motiva la } \\
\text { compra } \\
\text { 3. A veces hay que comprar algo para } \\
\text { sustituir otra cosa que ya no da el } \\
\text { servicio que se espera de ella } \\
\text { 2. Se necesita algo urgentemente en } \\
\text { un momento concreto y se compra lo } \\
\text { que está más a mano } \\
\text { 19. Compra algo simplemente para } \\
\text { sentirse más seguro } \\
\text { 1. Se tiene inevitablemente que llenar la } \\
\text { nevera. }\end{array}$ & $\begin{array}{l}.2169 \\
\\
.1753 \\
.1256 \\
.0960 \\
\\
.0916 \\
.0812\end{array}$ & $\begin{array}{l}0.000 \\
0.000 \\
0.000 \\
0.000 \\
\\
0.000 \\
0.000\end{array}$ \\
\hline $\begin{array}{l}\text { Necesidades } \\
\text { relativas } \\
\mathrm{R}^{2}=81.8 \%\end{array}$ & $\begin{array}{l}\text { 6. La gente compra simplemente } \\
\text { para sustituir cosas que ni tiene ni } \\
\text { nunca tendrá } \\
\text { 16. Se compra un producto para } \\
\text { dárselo a otra persona que } \\
\text { previamente le ha dado algo. } \\
\text { 13. El consumidor compra porque } \\
\text { se siente identificado con el target } \\
\text { del producto } \\
\text { 20. Un producto se compra } \\
\text { simplemente por darse el gusto de } \\
\text { hacerlo } \\
\text { 14. La gente compra lo que tienen }\end{array}$ & $\begin{array}{l}.1055 \\
.1009 \\
.0980 \\
.0952 \\
.0941 \\
.0880 \\
.0846\end{array}$ & $\begin{array}{l}0.000 \\
0.000 \\
0.000 \\
0.000 \\
0.000 \\
0.000 \\
0.000\end{array}$ \\
\hline
\end{tabular}




\begin{tabular}{|c|c|c|c|}
\hline & $\begin{array}{c}\text { también sus amigos } \\
\text { 12. El consumidor compra } \\
\text { simplemente un producto porque } \\
\text { cree que éste le va ayudar a sentirse } \\
\text { mejor que el resto } \\
\text { 5. Se compra un producto para } \\
\text { ganar en "prestigio" de cara a los } \\
\text { demás. }\end{array}$ & & \\
\hline $\begin{array}{c}\text { Esfuerzos } \\
\text { mercadológicos } \\
\mathrm{R}^{2}=74.9 \%\end{array}$ & $\begin{array}{l}\text { 10. El consumidor compra un } \\
\text { producto porque está de moda y todo } \\
\text { el mundo habla de él. } \\
\text { 17. Compra un producto porque } \\
\text { siente empatía por él, aun siendo } \\
\text { consciente de que su relación } \\
\text { calidad-precio no es la mejor } \\
\text { 4. Se compra un producto por su } \\
\text { exclusividad o porque sus } \\
\text { existencias son ya muy limitadas. } \\
\text { 7. Un producto a un precio } \\
\text { excepcionalmente bajo es casi } \\
\text { siempre un imán para el consumidor. } \\
\text { 15. Se compra un producto porque } \\
\text { se cree que haciéndolo está } \\
\text { contribuyendo a una buena causa } \\
\text { 9. Cuando compra por primera vez } \\
\text { un determinado producto, el } \\
\text { consumidor se suele decantar por la } \\
\text { marca más conocida, aquella que le } \\
\text { resulta más familiar. } \\
\text { 8. No se necesita en realidad el } \\
\text { producto, pero lo termina } \\
\text { comprando porque su relación } \\
\text { calidad-precio es inmejorable }\end{array}$ & $\begin{array}{l}.1472 \\
.1284 \\
.1104 \\
.0989 \\
.0935 \\
.0908 \\
.0624\end{array}$ & $\begin{array}{l}0.000 \\
0.000 \\
0.000 \\
0.000 \\
0.000 \\
0.000 \\
0.000\end{array}$ \\
\hline
\end{tabular}

\section{Conclusion}

En este estudio se ha analizado la importancia que los estudiantes universitarios en México dan a las 20 razones o factores de influencia propuestos por Eisenberg (Bryaneisenberg 2015), y que nosotros hemos sintetizado en tres dimensiones: necesidades innatas o absolutas, necesidades relativas y esfuerzos mercadológicos. Hemos encontrado que existe una relación (influencia) importante entre los esfuerzos de mercadeo desarrollados por las empresas y la decisión de compra del estudiante universitario, incluso superior a la influencia que pudieran ejercer sus necesidades innatas y sociales.

Sin embargo, para los practicantes de la mercadotecnia debe quedar muy claro que los hallazgos presentados en la tabla 3 confirman que los esfuerzos motivacionales, para atraer y retener a los consumidores, deben ser 
estratégicos al construirse de manera equilibrada, esto es, integrando también los factores de motivación innata y social.

Si bien los resultados se circunscriben a un segmento de mercado muy específico, consideramos que esta investigación abre la puerta a otros estudios que permitan analizar con mayor profundidad y detalle: (a) la validez y fortaleza de las 20 razones de influencia de Eisenberg, (b) su reclasificación entre las mismas dimensiones aquí propuestas, en las dimensiones clásicas (necesidades utilitarias y hedonistas) 0 en otras novedosas, (c) en otros segmentos del mercado, y (d) en categorías específicas de productos/servicios de consumo final.

\section{References:}

Abc (2015) consultado 18 de enero 2015 http://www.abc.es/tecnologia/redes/20141007/abci-razones-comprar-cosasnecesitamos-201410071316.html

Alonso, J. (2010), Comportamiento del consumidor, España, Esic p.56

ANUIES consultado 13 de abril de 2015 http://www.anuies.mx/anuies/instituciones-de-educacion-superior/

Arana, J., Meilán, J., Gordillo, F., \& Carro, J. (2010). Estrategias motivacionales y de aprendizaje para fomentar el consumo responsable desde la Escuela. R.E.M.E. (Revista Electrónica de Motivación y Emoción), Volumen XIII, No 35-36, págs. 19-39.

Attali, J. y Guillaume, M. (1974), Lántieconomique, París, Presses Universitaires de France

Beaton, C. (1990). El espejo de la moda. Barcelona: Parsifal.

Blackwell, R (2002), Comportamiento del consumidor, México, Thompson p.91

Boeree, G. (2003). Teorías de la personalidad, de Abraham Maslow Traducción: Rafael Gautier

Bryaneisenberg http://www.bryaneisenberg.com/about/bryan-eisenberg/ consultado 8 de enero 2015 block personal 20 razones por las que la gente compra

Cacioppo, J. T., Berntson, G. G., \& Klein, D. J. (1992). What is an emotion The role of somatovisceral afference, with special emphasis on somatovisceral "illusions." Review of Personality and Social Psychology, 14, 63-98, citado Palmero f., Fernández- Abascal, E. Martínez F., Chóliz M. (2002) Psicología de la motivación y la emoción, Mc Graw Hill, p.37

Cronbach, L. J. (1951). Coefficient alpha and the internal structure of tests. Psychometrika. 16, 297-334

Crompton, J.L. y Baker, D.A. (2000) Quality, satisfaction and behavioral intentions. Annals of tourism research 
Deckers, L. (2001) Motivation, Biological, Psychological and Environmental. Boston: Allyn and Bacon

Dickson, P.R., y Sawyer, A.G. (1990) The price knowledge and search of supermarket shoppers. Journal of Marketing, vol. 54, p.42-53

Eisenberg, B. (2002): The ROI of free. Consultado 8 de enero 2015 http://www.clickz.com/clickz/column/1699775/theroifree.

Eisenberg, B., Eisenberg, J. y Lisa, D. (2014) "Buyer Legends: The Executive Storyteller's Guide Hardcover - Bargain Price

Eisenberg, B., y Novo, J. (2002). The marketer's common sense guide to emetrics. Brooklyn (NY). Future Now.

Feldman, R. (2003). Introducción a la psicología. 4ta Ed. México. McGrawHill Interamericana. p.345

Ferre, J. (1997) Conducta del consumidor y del cliente España, Díaz de Santos, p.23

Galindo, A. (2010). Psicología del consumidor Mexicano. Segmento, Revista del Instituto Tecnológico de México, 48, 1- 4.Gil, A. (2004) Psicología económica en el comportamiento del consumidor Barcelona, UOC.

George, D. y Mallery, P. (1995). SPSS/PC+ step by step: A simple guide and reference. Belmont, USA: Wadsworth Publishing Company.

Gil, A. (2004) Psicología económica en el comportamiento del consumidor Barcelona, UOC.

Huitt, W. (2001) Motivation to learn: An overview. Educational Psychology Interactive, p. $12 . \quad$ Prensa Impresa http://impresa.prensa.com/vivir/Llenandovacioscosas_0_3303419842.html\#s thash.0pw85hLC.dpuf Consultado 17 de enero 2015 Kotler, P. (2000) Dirección de marketing México, Pearson, p.174

Kotler, P, \& Amstrong, G. (2001). Mercadotecnia. México: Pearson Educación.Lamb, Ch., Hair, J., y McDaniel, C. (2002), Fundamentos de Marketing, International Thomson Editores S.A., p.198-200 y 296-98.Lamb, Ch., Hair, J., y McDaniel, C. (2002), Fundamentos de Marketing, International Thomson Editores S.A., p.198-200 y 296-98.

Lambin, J.J., Gallucci, C., y Sicurello, C. (2009) Dirección de Marketing, Mc-Graw Hill, México p.68-70

Mareuvicentin.blogspot (2015). Consultado el 16 de febrero de 2015 http://mareuvicentin.blogspot.mx/2008/09/factores-psicolgicos-

delconsumidor.

Marketingdirecto (2011) consultado 18 de enero 2015 http://www.marketingdirecto.com/actualidad/checklists/20-razones-por-lasque-la-gente-compra/\#sthash.OFGax7Xa.dpuf

Maslow, A. H. (1954). Motivation and Personality. Estados Unidos: Harper \& Row. 
Maslow, A.H., y Hoffman, E.E. (1996). Future visions: The unpublished papers of Abraham Maslow. Sage Publications, Inc. New York.

Molla, A. (2006), Comportamiento del consumidor, Barcelona, UOC, p.44

Neoteo (2015) consultado 17 de enero 2015

http://www.neoteo.com/consumismo-realmente-necesitamos-tantas-

porquerias/

Palmero f., Fernández- Abascal, E. Martínez F., Chóliz M. (2002) Psicología de la motivación y la emoción, Mc Graw Hill, p.37

Petri, H.L. (1991). Motivation. Theory, Research, and Applications. Belmont, California: Wadsworth Publishing Company.

Poiret, P. (1930) En habillant l'epoque. París: Grasset

Pyme.lavoztx (2015) consultado 17 de enero 2015 http://pyme.lavoztx.com/la-importancia-del-reconocimiento-de-la-marca10803.html

Red académica (2015) consultado 13 de abril de 2015 http://redacademica.net/observatorio-academico/2012/10/03/la-educacion-superioren-mexico-2006-2012-un-balance-inicial/

Rivera, J. (2009) Conducta del consumidor, España, ESIC.

Rosa, J.J. (1977) Vrais et faux besoins, en Léconomique retrouvé, París Económica

Ruiz, S. (2006) Comportamiento de compra, España, Esic, p.36

Sergueyevna Golovina. N., y Mosher Valle, E. L. (2013). Teorías motivacionales desde la perspectiva de comportamiento del consumidor, Negotium, 9 (26), 5-18.Sexton, W.P., \& Contín, A. (1977) Teorías de la organización. Trillas.

Sheth, J.N., Newman, B.I., y Gross, B.L. (1991). Why we buy what we buy: a theory of consumption values. Journal of business research, 22(2), 159-70.

Stoner, J. (1996). La Motivación 6a. Edición. Editorial Pearson. México.

Vanhuele, M. y Drèze, X. (2002). Measuring the price knowledge shoppers bring to the store Journal of Marketing, vol. 60, p.91-104 